\title{
Pattern, Noise, Figure, Ground: Context and the Representation of the Auditory Object
}

\author{
REBECCA SMITH \\ University of Michigan
}

\section{INTRODUCTION}

Similarly to dirt, the term "noise" is often a moving target, defined in relation to what it is not. Noise is both subjective and objective, and is fundamentally reliant upon context for its definition. Specific to sound, the term "noise" can refer to anything from a style of music, to a physical entity with defined and quantifiable characteristics, capable of measurable effects on the human auditory apparatus. In a more colloquial or generalized usage, "noise" is often used to mean the unimportant clutter that gets in the way of the intentional or important content: a meaning similar to the "noise" of communications technology, itself originating in early 20th century theories of signal-to-noise ratio.

It is no surprise, then, that the term "noise" recurs in current conversations surrounding digital technology. In recent writing on the implications of data for architectural discourse and practice, both Mario Carpo and Benjamin Bratton evoke the concept of "noise" as a readily accessible shorthand for the condition that we increasingly find ourselves in; a condition wherein we have everything but cannot make sense of any of it. When Carpo describes Google's epistemological stance of "search, don't sort,"1 or Bratton describes the increasing significance of "addresses" rather than fixed positions, ${ }^{2}$ both describe something that we perhaps understand intuitively if not explicitly about the nature of data: that patterns and relationships are everything.

\section{RELATIONSHIPS AND DIFFERENTIATION}

"Noise" - and the patterns which define it - are the subject of the images shown in figure 1. The top row shows spectral visualizations: a technique which audiologists, sound engineers, and others use to analyze sound. Rather than waveforms, which show loudness alone, a spectral image plots the strength of the signal, using fast Fourier transforms, across time and the spectrum of human hearing. The reason for this has to do with the way that our bodies and minds process sounds, psychoacoustically (through the sensory and cognitive process of hearing). To put it simply, the way that we differentiate sounds from one another is by discerning patterns: either in time, in spectral / harmonic relationships, or both.

Spectral images are particularly useful because they explicitly visualize these relationships in figure / ground terms: the clearer a "figure" of a given sound in a spectrogram, the more distinct that sound will be to us, as listeners. This is illustrated by the images in the top row of figure 1 . None of these sounds are exceptionally "loud," but some are more perceptually present than others. In fact, while termed "noise," one example - white noise - is perhaps most familiar as a masking device: a sound which can cover up other, intrusive sounds for purposes of sleep or privacy. This masking quality translates into its spectral image: the energy of white noise is evenly distributed across both of the axes of the spectrogram. In figure-ground terms, there is no differentiation in either domain.

This tracks, perceptually, with our experience: it's relatively easy to let the sound of white noise fade into the background of our auditory environment. In contrast, alarm sounds are specifically engineered to be as annoying as possible: to grab our attention by recurring in a way that is consistently legible against whatever else is happening. This, too, tracks with the figure-ground image created by the spectral analysis: the sound of the alarm differentiates itself effectively against the background.

The critical point is that in both of these examples, what is most significant is not overall "loudness," but instead, the degree to which the sound is distinct from its context. This issue - of how entities are defined within auditory perception - is the first point of parallel between sound and data, for the purposes of this discussion. Perhaps most famously associated with the work of John Cage, the paradoxical mismatch between "silence" and "neutrality" is key, here. This is also where the analogy to data is strikingly clear: simply having "more" of something does not result in that "something" possessing greater significance or meaning; what is important is the pattern in context, rather than a freestanding or objective value.

This same tension exists in the spatial representation of environmental noise, such as the transportation noise map shown in figure 2. This kind of mapping is increasingly done predictively, through digital modelling techniques. However, there is an inherent challenge, in that these techniques of measurement - and the representational techniques associated with them-tend towards the objective and static, rather than the contextual and relational.

While overall measures of exposure to loudness are useful in determining hearing loss through physical damage to the human body, these same metrics can miss quality of life or "annoyance" noise. This is because these types of noises, as discussed above, have to be evaluated in relation to temporal patterns and spectral context. While there are researchers who are specifically working on this issue, mainly through developing methods which 

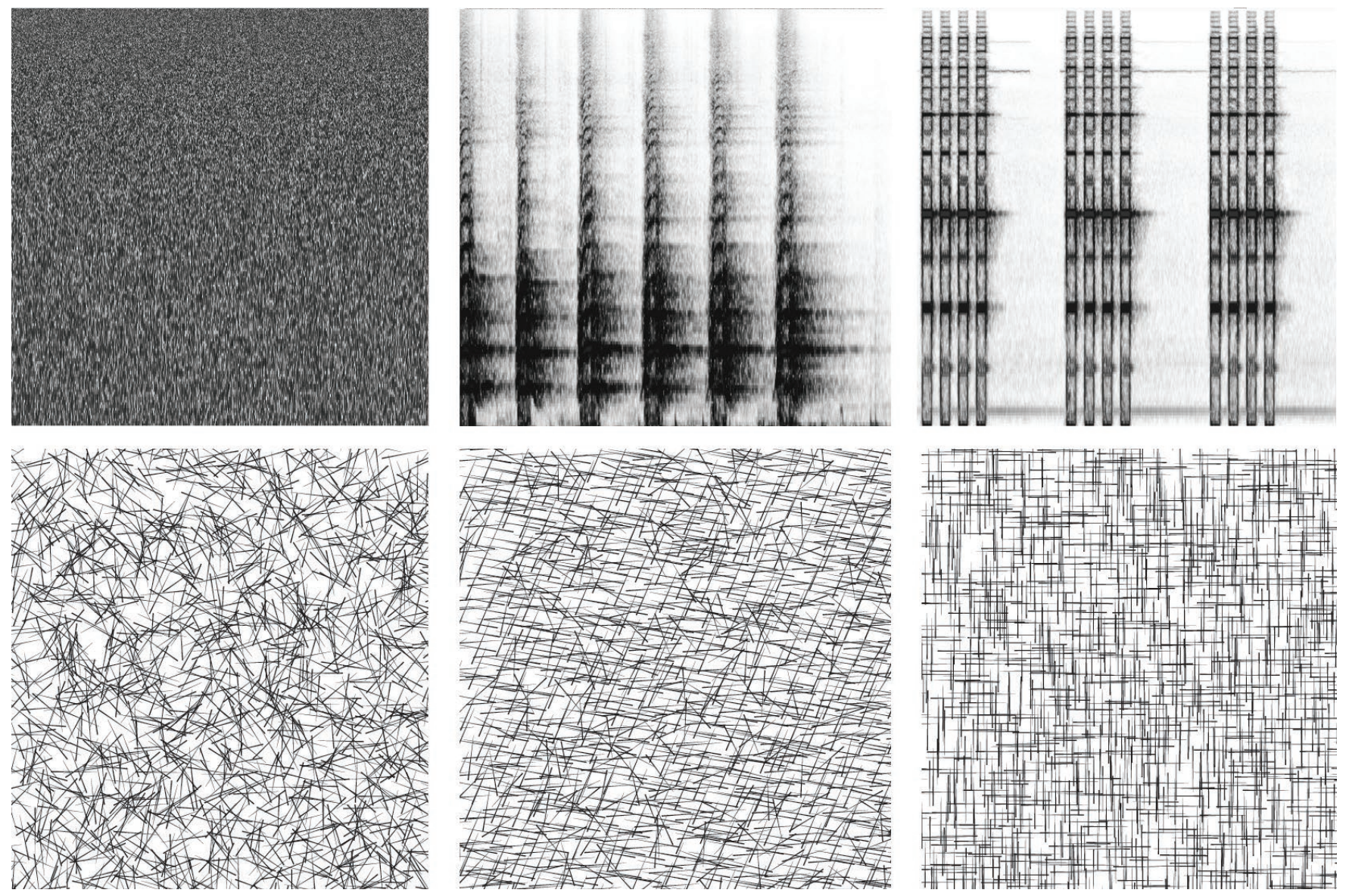

Figure 1. Top left to right: Spectral images of "noise:" white noise, dog barking, alarm clock. Bottom left to right: Parametrically generated patterns re-visualizing the same sounds.

quantify dynamic change and recurrence, spatial representations are still typically limited to those such as shown in figure $2 .^{3}$

Representational Studies: As an alternative to this, the drawings on the bottom row of figure 1 show representational studies which seek to re-visualize the information conveyed in the spectral images as a texture or pattern, to facilitate their combination with the conventions of architectural and/ or spatial representation. Given the emphasis on patterns and relationships within the perception of noise, the base form of the graphic language needed to be capable of showing difference without showing amount, able to show change without a hard boundary, and able to be aggregated and scaled. In order to function as a data-driven visualization, all attributes additionally needed to be controlled parametrically.

For these reasons, a skinny isosceles triangle was chosen as the base element. It was discovered to have an advantage over other simple geometry, because of its ability to show direction or grain - a useful characteristic in showing transition from one pattern to another. All of the drawings shown here are made from some aggregation of this form. What creates differentiation are the ways in which the disbursed elements relate to one another, through spacing, rotation, and size.
In this way, the representation aims to function similarly to the production of meaning within psychoacoustic perception: through ongoing changes in relationship between elements, as interpreted within patterns and sequences. Rather than the relationship of figure to ground, it is the relationship of pattern to pattern that becomes significant.

\section{PATTERNING, INFORMATION TECHNOLOGY AND PERCEPTUAL PSYCHOLOGY}

The difference between figure-ground and pattern, in many ways, underscores some of the larger theoretical and conceptual arcs accompanying the shift towards digital technology over the 20th and 21st centuries: a shift which, in a variety of fields, has signified a move away from singularity and towards multiplicity, away from binary and towards gradient, away from abstracted universality and towards contextualism, and in general, towards relational, contingent, and dynamic framings. While this shift, particularly in architecture, can be seen clearly in the confluence of post-modern and post-structural epistemological frameworks and digital technology in the 1990s, a similar interest in pattern, as aligned with communications technology and data, was present even in early cybernetic work. 
Reinhold Martin discusses the ways in which ideas of patterning and relational networks were prevalent topics of inquiry within early cybernetic thinking and research, as evidenced through a variety of intellectual modes of inquiry, aesthetic production, and procedural research, within the context of post-war media and technology. ${ }^{4}$ Martin describes Gyorgy Kepes' static visual representations of patterns, in Kepes' 1956 book "The New Landscape in Art and Science," as well as the sequential patterning of signals within contemporaneous approaches to theories of communications technology, specifically presenting the similarities between Kepes' focus on visual patterning and the interests of cybernetician Norman Weiner, for whom the significance of patterning was profound, as evidenced in his framing of human existence as "not stuff that abides, but patterns that perpetuate themselves."

Within this emphasis on pattern and relationships can be seen an early incarnation of one of the themes that would continue to accompany the growing influence of digital technology throughout the following decades: the understanding of the overall entity as determined by the relationships between smaller elements. The potential to map, explore or define the limits of these relationships, through recombinance and the determination of rulesets, was - and continues to be heavily enabled by the capabilities of digital technology. This can be seen within the early mainframe work of Christopher Alexander or lannis Xenakis in the 1960s and 70s, with their common interest in themes of stochasticism, probability, and

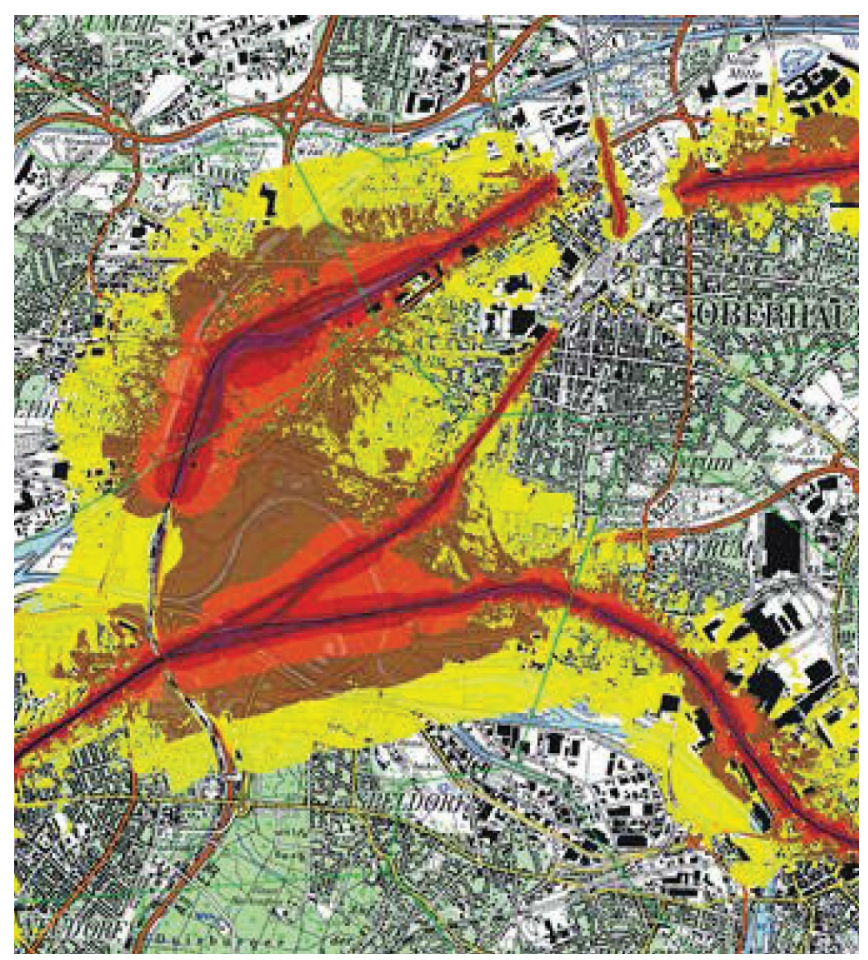

Figure 2. Transportation noise map based on predictive modelling. From www.soundplan.com/noise-control.html. process. It can also be seen within the wave of theoretical and critical approaches surrounding the rise of desktop computing in architectural culture during the 1990s, perhaps most explicitly in Stan Allen's framing of the field condition, which he defined as stressing "...not so much the forms of things as the forms between things." ${ }^{\prime \prime}$ In a very general sense, this lineage might suggest that the more information or data there is, the more relational the production of meaning becomes.

Arguably, our technologies of information have increasingly paralleled the ways in which our sensory and cognitive systems make meaning of our environment. Rather than discrete, abstracted chunks of meaning, we perceive our environment as a continually-evolving, fluid continuum. While many designers and technologists of the last and current centuries have been drawn to the increasingly complex ability, afforded by representational and communications technology, to translate, model, and emulate emergent natural systems, the ongoing goal of perceptual psychologists perhaps has been in the opposite direction: to decode and simplify this same type of complexity, in an effort to trace out commonalities, generalities, and rulesets across the variability of individual subjective experiences.

It follows that the relationship of pattern, meaning, and information has similarly been a recurrent area of interest for perceptual psychologists, beginning with Gestalt images representing figure-ground relationships. In contrast to this, psychologist J.J. Gibson's model of ecological perception presented an alternative which explicitly embraced "blurriness" as a valuable component of the perceptual information we receive from our encounter with the environment. For Gibson, as for the subfield of ecological psychoacoustics which evolved following his work, the edges of perception, and the processes through which sensory information shifts into - or out of - focus, is critical. ${ }^{7}$ This is even more pronounced when the ecological-perceptual framework is approached from a specifically psychoacoustic perspective, given the rapidly changing nature of the auditory environment. ${ }^{8}$

\section{PATTERN, TEMPORALITY AND SEQUENCE}

All perception is about pattern, as it involves the evaluation of entities in comparison to one another. Regardless of sensory modality, patterns are also always temporal to some degree, in that they are evaluated or understood sequentially; however, a key difference between vision and audition is how this temporal patterning occurs. This in turn relates to a subtle - but critical - epistemological or conceptual difference between the two.

When we refer to an "object" that is perceived through vision, we are generally referring to an object that is illuminated by light energy, rather than the light energy itself. This object might be changing position or form, but it does not rely upon dynamic change for its identity in a fundamental way. 


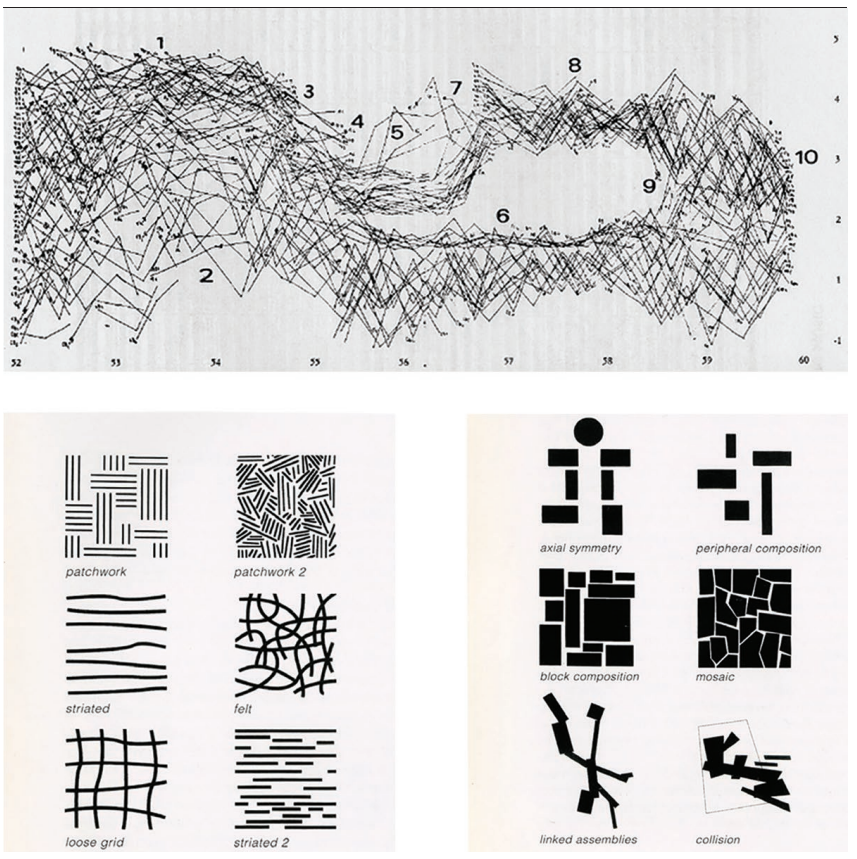

Figure 3. Top, lannis Xenakis, score for Prithprakta; bottom, Stan Allen, diagrams ("From Object To Field").

However, when we refer to a sound that we hear, the entity we are describing is sound energy itself: a rapidly fluctuating series of compressions and expansions which move through space and time, presenting themselves to our auditory apparatus as a quickly evolving, emergent entity. This is arguably an issue of temporal scale: while change, in an entropic sense, is a fundamental component of all aspects of our environment, auditory objects happen to be changing at a rate which, when encountered at the scale of human perception, emphasize issues of temporality, variability, and dynamism.

Timothy Morton discusses the interrelationship of temporality, human perception, and object identity at length in his discussion of what he terms the "hyperobject." Morton's hyperobjects are typically very large, as defined in both space and time (one key example is climate change). In a scalar sense, this moves in the opposite direction from the auditory object. However, his approach to space, time, and objecthood is useful to consider, particularly in relation to patterning, temporality, and human psychoacoustic perception. For Morton, patterning and fluctuation are a result of the limitations of our perceptual abilities. He argues that the appearance of fluctuations and patterns is only evidence of our limited ability to perceive hyperobjects, which do in fact have consistency, albeit out of the realm of our perception. Morton also applies this framing to the larger topic of the production of scientific knowledge, underscoring the interrelationship between the tools of quantification or representation, human perception, and the entity or phenomenon under study. In this, Morton is in line with many thinkers seeking to bracket or contextualize the pursuit of scientific objectivity, a position with relevance to practices of architectural representation specifically, as well as the datadriven paradigm more generally.

It is often assumed that the irregularity of individual subjective perception is less valid than the universalizing objectivity of standardized tools for the interpretation of the environment. This is borne out also in architectural representation: for example, when we look at a plan view of an enclosure, the burden of compensating for individual subject positions in space and time - not to mention the irregularity of individual perception, cognition, and subjectivity - is placed on the reader of the drawing. This is not inherently problematic, as representations of this kind are useful in a variety of contexts, but it does reinforce certain ideas about the continuity and consistency of space and time as normative - precisely the kind of normativity challenged by Morton. This same normativity supports the primacy of visual perception as a sensory modality, which in turn is baked into many standard architectural representational conventions. With this comes a set of assumptions about space and the organization of objects within it, assumptions which are productively challenged through a consideration of sound as a perceptual object.

As discussed above, object definition within auditory perception is determined through a combination of temporal and spectral patterning. Albert Bregman, a psychologist whose ecological approach to psychoacoustics follows in the lineage of Gibson, has done extensive experimental research to develop a framework translating Gestalt organizing principles for visual objects into similar principles applicable to auditory ones. In order to translate these principles from vision to audition, Bregman argues for the significance of time and frequency distribution (the two criteria plotted on the spectrograms shown earlier). He makes a critical point, particularly for architects: within auditory perception, time plays the organizing role for objects or entities that we typically associate with space in vision..$^{10}$ This is a concept with significant implications for architectural representational conventions regarding the evenness of space and relationships between objects within it. ${ }^{11}$

Representational Studies: The work shown in figure 4 shows the patterning technique discussed earlier, as applied to a short sequence of movement through an interior and exterior section of a large farmers market. Three different passes through the space are represented. The shifting grain in the pattern represents the changing distribution of spectral energy when passing under a large HVAC unit just inside of a set of double doors. The sound shifts from broadband (random rotation of elements, similar to the white noise example in figure 1), to a more tonal concentration of machine noise as the unit is approached. Once outside, the machine noise is no longer audible and is replaced by small incidental sounds. 

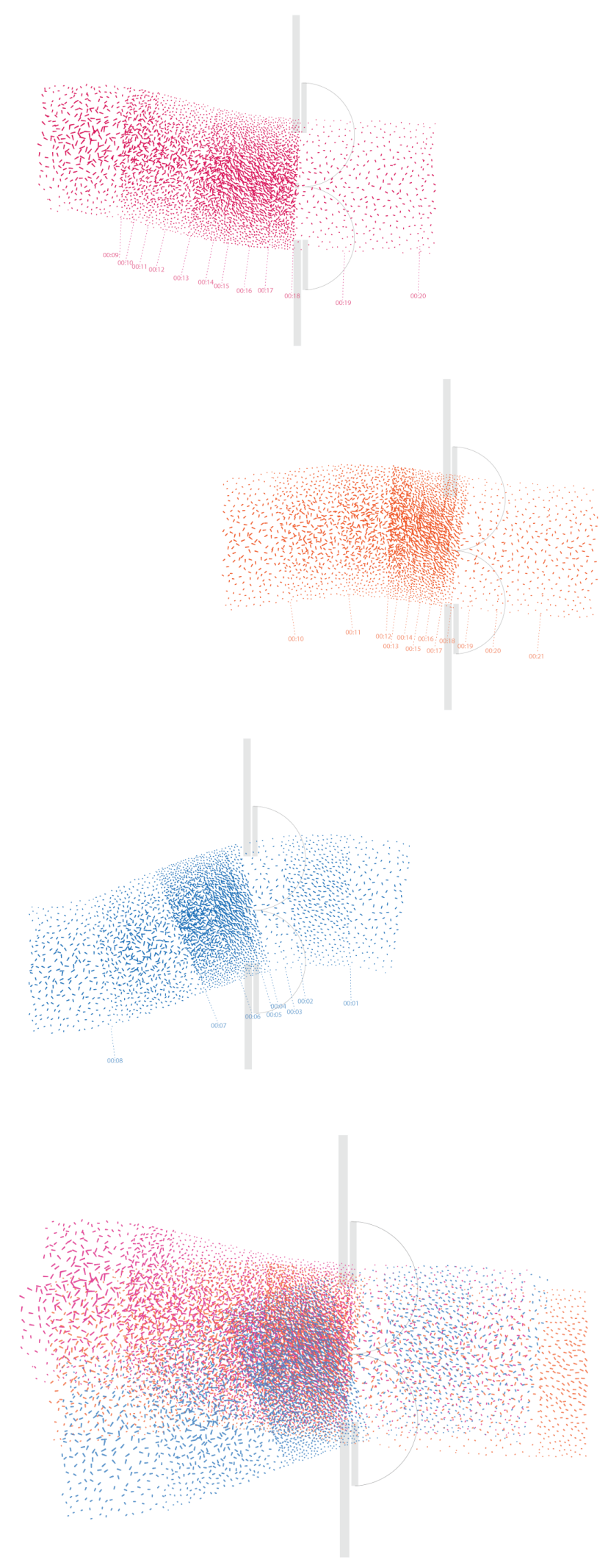

Figure 4. Representational studies showing three passes through the same transition from interior to exterior. Architecture remains consistent; sound is self-similar but variable.
As discussed above, the role of time in relation to the perception of sound and its representation is significant. Figures 4 and 5 explore some of the complications that this presents. In the same way that coordinated orthographic views are meant to work as a set, with neither considered more "correct" than the rest, these drawings are also coordinated representations: one privileging space, and the other privileging time. In figure 4, geometry describing one second segments is distorted to conform to a plan view, with space represented as even and consistent. Figure 5 inverts this relationship; instead of normalizing for space, these representations normalize for time, with the result that the representation of the architecture becomes deformed.

By showing three different passages through the same space, these drawings also introduce an additional complication related to the unavoidably temporal nature of the auditory object: variability. Both sets of drawings describe a set of self-similar- but not identical - encounters with sound in space and time. As a quickly fluctuating object, sound is constantly being refreshed or recreated in the mind of the individual subject, as it is evaluated in relation to its temporal and spectral context. This means that it is hyperspecific to an individual's position in space and time, which in turn results in multiple self-similar and variable representations. As discussed in relation to the significance of pattern in auditory perception, if these irregularities are smoothed out, much of the relevant information will be lost as well. While this kind of variability does present some challenges for the goals of standardization and universality often associated with architectural representational conventions, it also aligns with a number of contemporary perspectives within architectural discourse, particularly those dealing with the interrelationship between digital technology, representation, materiality, and subjectivity.

\section{SUBJECTIVE VARIABILITY, MATERIALITY, AND EMBODIED DIFFERENCE}

Concepts of variability and self-similarity have been taken up extensively within recent architectural discourse, particularly in relation to object, form, and digital production. One example of this is Mario Carpo's recent work which seeks to bracket "identicality" as a modern aberration; an anomaly bounded by artisanal idiosyncrasy on one side, and digital, scripted, meta-authorship on the other. Carpo argues that material production, formerly oriented towards standardization for the purposes of profit, has been re-oriented, in the contemporary context, towards the opposite direction: customization. ${ }^{12}$ Carpo stops just short of further political implications of the kind of variability he describes, specifically the application of this variability to the subject. For many theorists dealing with digital technology, however, variability is most significant precisely when applied to subjectivity and subject / object relationships. 
Antoine Picon argues that the digital context produces a new materiality, one which brings together opposite extremes of abstract and concrete, virtual and physical, and increasingly decreases the distance between representation and object. ${ }^{13}$ Picon discusses variability in a social and contingent sense: through an emphasis on the increasing significance of "occurrences, events, and scenarios." ${ }^{14}$ Picon's framing of materiality is not limited to physical, visually apprehensible objects as defined in a strict sense, but rather, implies an ongoing set of relations between physical form, representation, affect and subjectivity.

Picon takes a wide view of materiality, which he frames (with some interesting parallels to Gibson) as embodied encounter with environment . Caroline Jones, too, argues for the significance of the body itself as a site of encounters between perception, technology, affect, and cognition. ${ }^{15}$ Jones presents the site of the body as both requiring and affording a grounding of the virtual. This framing is particularly useful as it uses embodiment to simultaneously hybridizes both subject / object and material / immaterial dichotomies.

Perspectives on embodiment and subjectivity from other disciplines also offer applicable frameworks on variability, particularly if architectural representation is considered in terms of epistemology and communication. In this context, Steven Feld, a linguistic anthropologist and ethnomusicologist, addresses the tensions between the specificity of individual perception and the more universalizing goal of communication. Feld uses his term "acoustemology" to frame the perception of sound as a kind of relational, contextual, and experiential form of knowing. ${ }^{16} \mathrm{He}$ describes acoustemology as simultaneously locating the specificity of perceptual ontology within the realm of the individual, while also allowing this to fade into a larger social sphere, where it becomes a communally held form of collective knowledge which transcends the interiority of the individual subject.

In unpacking this idea, Feld utilizes the "situated knowledge" of Donna Haraway, as a framework with which to address the inherent conflict of the subject-privileging epistemological practice which he advocates. Haraway does not address hearing, sound, or psychoacoustics, but rather, presents the merits of "situated" or "embodied" knowledge, specifically as undertaken within the perspective of a feminist critique of science. ${ }^{17}$ As framed by Haraway, this critique is, by default, pushed into an embodied position, as it stems from the nonnormative position of the female body within the dominant scientific paradigm of the 1980s. As such, her position is explicitly variable. Haraway's overall argument is that claims to total or complete impartiality or objectivity are false and need to be interrogated, as only "partial" forms of "situated" knowledge (those which acknowledge their position and recognize the production of knowledge as an ongoing, participatory process), produce valid or legitimate results.
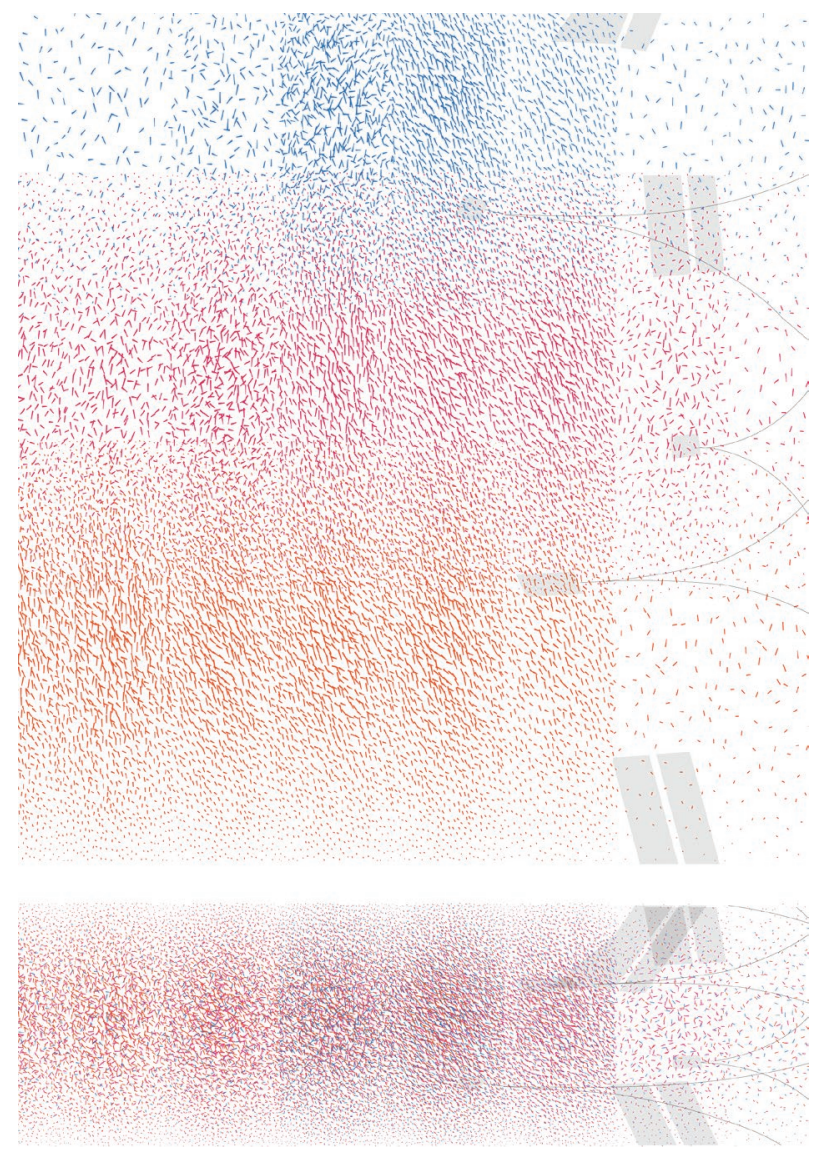

Figure 5. Representational studies showing three passes through the same transition from interior to exterior, reorganized in terms of time.

\section{CONCLUSION}

Feld and Haraway both present frameworks legitimizing the production of knowledge from a localized, embodied subject position, and seek to leverage these against dominant power structures in their respective disciplines. For both, the role of "context" is central in negotiating an area of subjective legitimacy. To return to the themes introduced at the beginning of this discussion, rather than isolated, objective "figures" of knowledge, both advocate for a relational understanding, one more akin to that of figure to ground.

However, this kind of relational framing requires an extra level of unpacking when considered from an architectural perspective, one which encompasses the complex set of factors discussed by Carpo, Picon, and Jones. For most humanities-based disciplines, "context" is an abstract and non-material phenomenon. For architects, however, "context" has inherently spatial and material dimensions, ones which we are used to describing in precisely scaled, measured and universal terms, through representational conventions which tend towards the objective and standardized. 
This is where the potential for representational techniques of variability and non-specificity, as enabled through datadriven techniques, might be considered as applicable to the challenge of showing, in spatial, material, and object terms, what both Feld and Haraway describe in textual ones: the simultaneity of both subjective and objective environmental reality, a scenario where interior subjectivity fades at times into a larger, expanded, collective. Rather than requiring an "either / or" framing in relation to questions of subjectivity and objectivity, could layering and aggregative techniques, as enabled by a data-driven approach, instead allow for a "both / and" approach? Given the social, cultural and epistemological values inherent in knowledge formations and representational practices oriented towards the validity of objectivity, what are the implications of alternative representational techniques, ones which utilize aggregation and visualization to achieve ambiguity, multiplicity and variability, rather than standardization and universality? What might the legitimization of subjective difference, tendencies and commonalities, look like?

\section{ENDNOTES}

1 Mario Carpo, The Second Digital Turn: Design Beyond Intelligence (Cambridge, MA: The MIT Press, 2017), 25.

2 Benjamin H. Bratton, The Stack: On Software and Sovereignty (Cambridge, MA: The MIT Press, 2015), 191-204.

3 G. Memoli and G. Licitra, "From Noise To Annoyance Mapping: A Soundscape Approach," in Noise Mapping in the EU Models and Procedures, ed., Gaetano Licitra (Boca Raton: CRC Press; Taylor \& Francis Group, 2013).

4 Reinhold Martin, The Organizational Complex : Architecture, Media, and Corporate Space (Cambridge, MA: The MIT Press, 2003).

5 Martin, Organizational Complex, 24.

6 Stan Allen, "From Object To Field," Architectural Design 67, no. 5/6 (1997): 1.

7 Gibson details the specific point regarding blurriness in J. J. Gibson, "Visualizing Conceived as Visual Apprehending without Any Particular Point of Observation," Leonardo 7, no. 1 (1974): 41-42; as well as J. J. Gibson, "The Optical Expansion Pattern in Aerial Locomotion," The American Journal of Psychology 68, no. 3 (1955): 480-84. A more comprehensive discussion of Gibson's approach to perception overall can be found in in J.J. Gibson, The Senses Considered As Perceptual Systems (Boston, MA: Houghton-Mifflin, 1966).

8 For more on this, see John G. Neuhoff, Ecological Psychoacoustics (Amsterdam; Boston: Elsevier Academic Press, 2004).

9 Timothy Morton, Hyperobjects: Philosophy and Ecology after the End of the World (Minneapolis: University of Minnesota Press, 2013).

10 Albert S. Bregman, Auditory Scene Analysis: The Perceptual Organization of Sound (Cambridge, MA: The MIT Press, 1990).

11 For more on this concept, see also David Van Valkenburg and Michael Kubovy, "From Gibson's Fire to Gestalts: A Bridge-Building Theory of Perceptual Objecthood," in Ecological Psychoacoustics, ed., John G. Neuhoff (Leiden, Netherlands: Brill, 2004).

12 Mario Carpo, The Alphabet and the Algorithm (Cambridge, MA: The MIT Press, 2011), 41.

13 Antoine Picon, "Architecture and the Virtual: Towards a New Materiality," in Constructing a New Agenda, Architectural Theory 1993-2009 (New York: Princeton Architectural Press, 2004), 285.

14 Antoine Picon, Digital Culture in Architecture: An Introduction for the Design Professions (Basel: Birkhäuser, 2010), 13.

15 Caroline A. Jones, "Introduction" and "The Mediated Sensorium," in Sensorium: Embodied Experience, Technology, and Contemporary Art, eds., Caroline A. Jones and Bill Arning (Cambridge, MA: The MIT Press, 2006).

16 Steven Feld, "Acoustemology," in Keywords in Sound (Durham: Duke University Press, 2015)

17 Donna Haraway, "Situated Knowledges: The Science Question in Feminism and the Privilege of Partial Perspective," Feminist Studies 14, no. 3 (1988): 575-99. 